\title{
Electromagnetically induced left-handedness in a dense gas of three-level atoms
}

\author{
M. Ö. Oktel* \\ Department of Physics, Bilkent University, 06800 Bilkent, Ankara, Turkey \\ Ö. E. Müstecaplığlu ${ }^{\dagger}$ \\ Department of Physics, Koç University, Rumelifeneri Yolu, 34450 Sariyer, Istanbul, Turkey
}

(Received 9 June 2004; published 5 November 2004)

\begin{abstract}
We discuss how a three-level system can be used to change the frequency-dependent magnetic permeability of an atomic gas to be significantly different from 1 . We derive the conditions for such a scheme to be successful and briefly discuss the resulting macroscopic electrodynamics. We find that it may be possible to obtain left-handed electrodynamics for an atomic gas using three atomic levels.
\end{abstract}

DOI: $10.1103 /$ PhysRevA.70.053806

\section{INTRODUCTION}

Changing the propagation properties of light by designing a novel material is of interest both from a basic science point of view, and for technological applications. Recent advances such as slowing down [1] or stopping light [2] or left-handed metamaterials [3] promise advances in fields ranging from optics [4] to quantum computation [5]. It is desirable to find materials in which electromagnetic waves exhibit novel behavior, and there is a flurry of activity both theoretically and experimentally in this direction.

The macroscopic electromagnetic (em) properties of a medium are characterized by the frequency-dependent dielectric constant [6]

$$
\vec{D}=\varepsilon(\omega) \vec{E}
$$

and the magnetic permeability

$$
\vec{B}=\mu(\omega) \vec{H} .
$$

The speed of an em wave of frequency $\omega$ in this medium is given by

$$
v=\frac{c}{\sqrt{\mu(\omega) \varepsilon(\omega)}}
$$

where $c$ is the speed of light in vacuum. The index of refraction is then $n=\sqrt{\mu(\omega) \varepsilon(\omega) \text {. }}$

The dielectric constant of the medium shows large variations near a resonance, i.e., when the frequency of the external field is near an internal state transition. This makes it easy to change the refractive index of a medium by properly tuning the frequency of the em wave to just above or below a transition. Recently this fact was employed combined with quantum coherent effects to get very high refractive indices in atomic gases [1,7].

Although $\varepsilon(\omega)$ can change appreciably for a gas of atoms at optical frequencies, the magnetic permeability $\mu(\omega)$ is always very close to its free space value. One can give argu-

\footnotetext{
*Electronic address: oktel@fen.bilkent.edu.tr

†Electronic address: omustecap@ku.edu.tr
}

PACS number(s): 42.50.Gy, 32.80.Pj, 03.75.Nt, 42.25.Bs

ments in classical electrodynamics to explain this [8], or understand it in terms of atomic transitions as follows. The magnetic field component of an em wave couples to the atom much more weakly than the electric field component. The magnetic coupling to an atom is proportional to the Bohr magneton $\mu_{B}=e \hbar / 2 m_{e} c=\alpha e a_{0}$, while the electric coupling is $e a_{0}$. The fine structure constant $\alpha \simeq 1 / 137$ also shows itself in the induced magnetic dipole moment. Overall the effect of an em wave on magnetic permeability is $\alpha^{2}$ weaker than its effect on the electric susceptibility. Another important fact is that magnetic dipole transitions are allowed only between states that have the same radial wave function, and generally two such states are not separated by optical frequencies in energy.

Now that it is hard to get $\mu(\omega)$ to be different from 1 , we need to question why it is important to have another value for it. After all it seems from Eq. (3) that all the optical properties of the medium depend on the product $\mu(\omega) \varepsilon(\omega)$. The answer to this question is that the refractive index alone does not completely represent the medium [9]. One can imagine two media, one with $\varepsilon_{1}(\omega)>0, \mu_{1}(\omega)>0$ and $\epsilon_{2}(\omega)=-\epsilon_{1}(\omega), \mu_{2}(\omega)=-\mu_{1}(\omega)$. They would have the same refraction index, but quite different optical properties. Materials with both $\varepsilon<0$ and $\mu<0$, are called left-handed materials, named for the parity of the coordinate frame formed by $\{\vec{E}, \vec{H}, \vec{k}\}$. The optical properties of left-handed and righthanded materials differ mainly because the Poynting vector points opposite to $\vec{k}$ in left-handed materials. The most remarkable change happens at the interface between a lefthanded material and a right-handed material, where the usual Snell refraction law gets a sign change. In addition to the inverse Snell law, reverse Čerenkov radiation and a reverse Doppler shift would also be possible in such materials [9].

Left-handed artificial materials in the microwave region have recently been built [10] by assembling a composite lattice of metallic split ring resonators and metallic wires [11], with periodicity much smaller than the wavelength of the electromagnetic field, or using anomalous propagation properties of light in a photonic crystal, with periodicity on the order of the wavelength of the electromagnetic radiation [12]. All such systems (called metamaterials) require delicate manufacturing of spatially periodic structures. In the micro- 
wave region, improvements of focusing, filtering, and steering properties of microwaves would be useful for many practical applications. Similar improvements would also be valuable for applications operating at optical frequencies. In this paper we examine the case of an atomic gas without any spatial periodicity that could exhibit behavior similar to metamaterials at optical frequencies.

We have remarked that the magnetic dipole response to an oscillating magnetic field is smaller by a factor of $\alpha^{2}$ compared to the electric dipole response to an oscillating magnetic field. In an em wave the $\vec{E}$ and $\vec{B}$ fields are always perpendicular to each other and are always in phase. If one can get the atom to respond to an electric field $\vec{E}$ with a magnetic moment $\vec{\mu}$ perpendicular to it, and in phase with it, one can effectively think that the magnetic dipole moment is induced by the magnetic field of the em wave. Thus, it is possible to get a magnetic response which is only $\alpha$ times smaller than the electric response. Such a response enables one to achieve a regime where the propagation properties of light are significantly different.

The aim of this paper is to explore the feasibility of this idea of modifying the magnetic permeability of an atomic gas electromagnetically. To this end, we introduce a model system in the next section and find the necessary conditions for the applicability of our scheme. In Sec. III we present the results of our calculations for two different parameter regimes, a dilute gas and a dense gas. We then go on to discuss the consequences of our results for experiments. Finally, we give a summary of our results and conclusions in Sec. IV.

\section{MODEL SYSTEM}

In this section, we construct a model system for which the magnetic permeability can be optically modified. We also describe the scheme for modification in detail, and discuss its limitations.

One can readily conclude by parity arguments that it is not possible to get a magnetic response to an electric field if only two states are involved. An electric field causes transitions to states which are of opposite parity to the ground state, and such states do not have a magnetic dipole matrix element with the ground state. To overcome this difficulty, we use a three-level scheme, similar to the one used in electromagnetically induced transparency (EIT) $[7,13]$ where an optically thick substance is made transparent and exhibits a large dispersive response to the external field close to atomic resonance.

The particular EIT scheme here serves several useful features required for left-handedness, such as being dispersive and exhibiting resonance phenomena. EIT materials do not suffer from linear absorption at resonance. They exhibit small transmission losses even at high densities. As a consequence of the resonance, the EIT medium stores a large amount of energy over the cycles of interaction, leading to a strong material response. In order to have a negative electric and magnetic material response, we need both the macroscopic polarization and magnetization of the material to become simultaneously so strong that they would be immune even to sign changes of the applied fields. For a weak probe

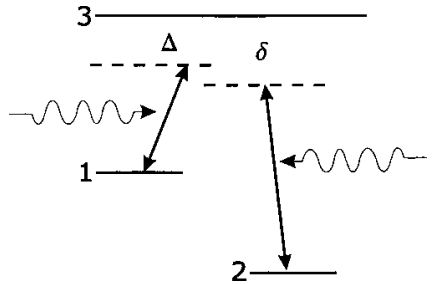

FIG. 1. Three-level atom interacting with the probe and the coupling fields in a $\Lambda$ scheme as described in the text.

beam, EIT cannot achieve this feat single-handedly. By considering a dense medium, with many particles within a cubic resonance wavelength, we let the local fields in the substance help to enhance the material responses. Indeed, we see that circularly polarized probe electric field, under EIT conditions, together with the help of the Lorentz-Lorenz local field contribution, could maintain strong local currents that could give rise to large enough magnetization, insensitive to sign changes of the probe magnetic field. At the same time, the electric response also becomes negative. The remainder of the section presents the mathematics behind these ideas as well as the conditions of their applicability.

We require the three states to have the following nonzero matrix elements:

$$
\begin{aligned}
& \langle 1|e \vec{r}| 3\rangle \neq 0, \\
& \langle 2|e \vec{r}| 3\rangle \neq 0, \\
& \langle 1|\vec{\mu}| 2\rangle \neq 0 .
\end{aligned}
$$

Here $\vec{\mu}$ is the magnetic dipole moment operator given by

$$
\vec{\mu}=\frac{\mu_{B}}{\hbar}\left(g_{L} \vec{L}+g_{S} \vec{S}+g_{I} \vec{I}\right),
$$

where the first two terms are the magnetic moments due to the electronic orbital angular momentum $L$ and spin angular momentum $S$, while the last term is the contribution of nuclear spin angular momentum $I$. The coefficients are $g_{L}$ $=1$ and $g_{S}=2$ (within a small $0.1 \%$ correction found by quantum electrodynamical calculations). The nucleon magneton is about 1800 times smaller than the Bohr magneton. Typical nuclear magnetic moments are about 1000 times smaller than their electronic counterparts and hence usually negligible. If the Hamiltonian is parity invariant, we can choose all the states to be eigenstates of the parity operator $\mathcal{P}$. To satisfy the requirement (4) one should have

$$
\langle 1|\mathcal{P}| 1\rangle=\langle 2|\mathcal{P}| 2\rangle=-\langle 3|\mathcal{P}| 3\rangle .
$$

We assume that the states $|2\rangle$ and $|3\rangle$ are coupled with an intense coherent beam while a weak probe beam will excite transitions between $|1\rangle$ and $|3\rangle$. We will investigate the dielectric permittivity and magnetic permeability of a medium consisting of such atoms as a response to the probe beam.

Such a system of a three-level atom interacting with those two optical fields in a $\Lambda$ scheme as depicted in Fig. 1 is described by a Hamiltonian in the form 


$$
H=H_{0}+H_{1},
$$

where

$$
H_{0}=\sum_{i=1}^{3} \hbar \omega_{i} R_{i i}
$$

and

$$
H_{1}=-\frac{\hbar}{2} \sum_{i=1,2}\left(\Omega_{i} e^{-i \nu_{i} t} R_{3 i}+\text { c.c. }\right) \text {. }
$$

Here, $\hbar \omega_{i}$ are the energy levels of a free atom, and $R_{i j}$ $=|i\rangle\langle j|$ are atomic projection operators. The interaction Hamiltonian is written under the electric dipole approximation. The Rabi frequencies associated with the optical transitions are defined by

$$
\Omega_{i}=\frac{\vec{d}_{3 i} \cdot \vec{E}_{i}}{\hbar}
$$

where the $\vec{E}_{i}$ stands for the complex amplitude of the positive frequency component electric field of the probe laser. The electric dipole operator is expressed as

$$
\vec{d}_{3 i}=e\langle 3|\vec{r}| i\rangle .
$$

Within the semiclassical theory of optical interactions, the density matrix of the system evolves according to the Liouville equation

$$
\frac{d \rho}{d t}=-\frac{i}{\hbar}[H, \rho]-\frac{1}{2}\{\Gamma, \rho\}
$$

We assume a diagonal relaxation matrix $\langle i|\Gamma| j\rangle=\gamma_{i} \delta_{i j}$, and choose the relaxation rates for off-diagonal elements of the density matrix as $2 \gamma_{i j}=\gamma_{i}+\gamma_{j}$.

Here we remark that the density matrix equations (optical Bloch equations) $[7,14]$ could be written more generally for a dense medium in terms of the total local field. Within the linear response theory, and assuming the material under consideration is linear, we will take into account the LorentzLorenz correction after determining the dilute material response as usual [15].

By considering the above equation for each component of the density matrix, the formal solution for $\rho_{21}(t)$ can be written as

$$
\rho_{21}(t)=\frac{i}{2} \Omega_{2}^{*} e^{i \nu_{2} t} \int_{0}^{\infty} \rho_{31}\left(t-t^{\prime}\right) e^{-i\left[\left(\omega_{21}+\nu_{2}\right)+\gamma_{21}\right] t^{\prime}} d t^{\prime},
$$

which leads to

$$
\begin{aligned}
\dot{\tilde{\rho}}_{31}= & -\left(i \Delta+\gamma_{31}\right) \widetilde{\rho}_{31}-\frac{i}{2} \Omega_{1}\left(\rho_{33}-\rho_{11}\right) \\
& -\frac{\left|\Omega_{2}\right|^{2}}{4} \int_{0}^{\infty} \tilde{\rho}_{31}\left(t-t^{\prime}\right) e^{-i\left[(\Delta-\delta)+\gamma_{21}\right] t^{\prime}} d t^{\prime} .
\end{aligned}
$$

Here, we introduced a slow variable $\tilde{\rho}_{31}=\rho_{31} e^{i \nu_{1} t}$, detuning of the probe beam $\Delta=\omega_{31}-\nu_{1}$, and detuning of the driving beam $\delta=\omega_{32}-\nu_{2}$.
The effect of a weak probe field on the system can be treated perturbatively. To first order in the probe field amplitude, we find

$$
\tilde{\rho}_{31}=\frac{i}{2} \Omega_{1} \frac{\left[i(\Delta-\delta)+\gamma_{21}\right]}{\left(i \Delta+\gamma_{31}\right)\left[i(\Delta-\delta)+\gamma_{21}\right]+\left|\Omega_{2}\right|^{2} / 4} .
$$

The positive frequency component of the complex induced electric dipole moment of the atom is given by $p_{i}=d_{13}^{i} \rho_{31}$, which is related to the complex atomic polarizability tensor $\alpha$ as $p_{i}=\alpha_{i j} E_{1 j}$. We adopt the summation convention, in which summation over a repeated index is implied.

For the macroscopic polarization we have to take into account local field effects which lead to the ClausiusMossotti [6] relation between the polarizability and the susceptibility $\chi_{e}$. For a small enough concentration $N$ of atoms $\chi_{e}=N \alpha \epsilon_{0}$ holds. Using $P_{i}=\epsilon_{0} \chi_{e}^{i j} E_{1 j}=d_{13}^{i} \rho_{31}$, we identify the complex electric susceptibility tensor for a gas of such threelevel atoms with concentration $N$ to be $[7,14]$

$$
\begin{gathered}
\alpha^{i j}=\frac{i}{2} \frac{d_{13}^{i} d_{31}^{j}}{\gamma_{31} \hbar \epsilon_{0}} \frac{1}{D}, \\
D=-\frac{\Delta}{\gamma_{31}}-i\left(1+\frac{\Omega_{2}^{2}}{4 \gamma_{31}\left[i(\Delta-\delta)+\gamma_{21}\right]}\right), \\
\chi_{e}=N \alpha\left(1-\frac{N}{3 \epsilon_{0}} \alpha\right)^{-1} .
\end{gathered}
$$

The complex dielectric permittivity tensor can be similarly constructed via $\epsilon_{i j}=\epsilon_{0}\left(\delta_{i j}+\chi_{e}^{i j}\right)$. We observe that this contributes to the complex permeability tensor of the system. It should be noted that for $\delta=0$ and for small $N$, we recover the well-known results for an electromagnetically induced transparent system. Now, using the equation

$$
\dot{\rho}_{21}=-\left(i \omega_{21}+\gamma_{21}\right) \rho_{21}+\frac{i}{2} \Omega_{2}^{*} \tilde{\rho}_{31} e^{i\left(\nu_{2}-\nu_{1}\right) t},
$$

we deduce the relation

$$
\tilde{\rho}_{21}=\frac{i}{2} \frac{\Omega_{2}^{*}}{i(\Delta-\delta)+\gamma_{21}} \widetilde{\rho}_{31}
$$

for the new variable $\tilde{\rho}_{21}=\rho_{21} \exp i\left(\nu_{1}-\nu_{2}\right) t$.

We can now calculate the induced magnetic dipole moment of the atom using

$$
\langle\vec{\mu}\rangle=\operatorname{Tr}(\rho \vec{\mu})
$$

where $\vec{\mu}=\mu_{B} \vec{L} / \hbar$ is considered for the magnetic dipole operator by assuming the contributions from nuclear spin are negligible. The electronic spin part is omitted for simplicity.

As the lower levels are of opposite parity to the upper level, the only nonvanishing contribution may arise if the lower levels are of the same parity. In this case we get $\langle\vec{\mu}\rangle$ $=\rho_{21} \vec{\mu}_{12}+$ c.c., which gives

$$
\langle\vec{\mu}\rangle=-\frac{\Omega_{1} \Omega_{2}^{*} \vec{\mu}_{12} \exp \left(i\left(\nu_{1}-\nu_{2}\right) t\right)}{4\left(i \Delta+\gamma_{31}\right)\left[i(\Delta-\delta)+\gamma_{21}\right]+\left|\Omega_{2}\right|^{2}}+\text { c.c. }
$$


In order to describe the atomic response to the magnetic field component of the probe field, we let the induced magnetic dipole of the atom oscillate in phase with the probe beam. This is achieved when $\nu_{1}-\nu_{2}= \pm \nu_{1}$. Setting aside the static field solution we consider the case of $\nu_{2}=2 \nu_{1}$. The other possibility, $\nu_{2}=0$, would be the case of a static electric field as the coupling field. This should be separately discussed as it is necessary to examine the Stark shifts of the levels and modify the present theory accordingly. The driving field is taken to be resonant with $\omega_{32}$ when the probe is resonant with $\omega_{31}$ so that $\delta=2 \Delta$, which puts a constraint on the three-level system as

$$
\omega_{32}=2 \omega_{31} .
$$

This constraint is, however, a major obstacle in realizing the predicted effects here at a realistic experimental setting as it is not straightforward to find a system with two states, which have a matrix element of $\vec{\mu}$ between them and at the same time have energy difference in the optical range. This is mainly due to the fact that $\mu$ is an angular operator and the two states involved should have the same radial wave functions to give a nonzero matrix element. One can imagine some external magnetic field adjusting the separations to give the necessary energy conditions. However, for an atomic system to get splittings in the optical regime, the external field would be impractically large. To our knowledge, currently there is no atomic gaseous system that satisfies this constraint and hence can be used to check our $\mu$ modification scheme directly. However, in the following paragraphs we would like to identify some candidate systems, where two states which have a splitting in the optical range and a magnetic dipole matrix element between them can be created.

As far as atomic gases are concerned, the best option seems to be to take two states which have the same $L$ value but which are split due to $L-S$ coupling, to be the states $|1\rangle$ and $|2\rangle$ and try to get a third level of opposite parity to satisfy the energy condition. Another direction to proceed would be to consider all our discussion for an atomic system under high electric field. In that case, it will not be too hard to get to fields which give shifts on the order of optical frequencies; however, one must carefully do the preceding analysis again, taking into account the effect of a static electric field on all three states. One other idea would be to consider systems where $\mu$ is not an angular operator, such as molecular gases. For such systems, one must not only identify states that satisfy the above constraint, bust also make sure that these states live long enough for a three-level coherent scheme to be successfully used.

One other, perhaps more promising, experimental direction is to apply our scheme to a solid state system. Coherent three-level effects [16] such as EIT have been demonstrated in quantum well structures [17], leading to exciting applications such as lasing without inversion [18], or slow light [19]. In these systems, the three states involved can no longer be simply thought of as single particle states, but generally are collective many-body states. Nevertheless, in a well prepared system, these three states are isolated sufficiently to allow for coherent effects. The major obstacle for application of our scheme to atomic gases is not present in these systems, as the energy splittings of the states depend on parameters of the quantum well, and can be designed to satisfy our constraint. Similarly, the energy spectrum of quantum dots can be designed to give three states that satisfy our constraint [20].

The application of our scheme for $\mu$ modification to any system, whether atomic gas or solid state, is possible if one can find three states which satisfy two constraints. The first constraint Eq. (4) concerns the matrix elements between the two states, while the other, Eq. (20), determines the splitting of the three states. We hope that either in the systems suggested above or in some other system three such states can be found.

We assume these conditions are satisfied with our hypothetical model atom and proceed by writing the product $\Omega_{1} \vec{\mu}_{12}$ explicitly, so that we can examine the directional character of the magnetic response of the atom to the probe field. The electric dipole of the probe transition and the magnetic dipole of the lower levels are combined through a tensor product relation such that

$$
\Omega_{1}\left(\vec{\mu}_{12}\right)_{i}=\frac{E_{1}}{\hbar} \sum_{j} \nu^{i j} \epsilon_{1 j}
$$

where we introduce

$$
\nu^{i j}=\left\langle 1\left|\mu_{i}\right| 2\right\rangle\left\langle 3\left|d_{31}^{j}\right| 1\right\rangle .
$$

The tensor $\nu$ demonstrates the combined effect of the electric and magnetic field components of the optical field on the directional character of the magnetic response of the medium.

To calculate the induced magnetic dipole moment matrix elements, it is convenient to consider the angular momentum basis in which we can also calculate the elements of the electric dipole moment using the Wigner-Eckart theorem. Let us identify the states as

$$
\begin{gathered}
|1\rangle \equiv|n, l, m\rangle, \\
|2\rangle \equiv|n, l, m-1\rangle, \\
|3\rangle \equiv\left|n^{\prime}, l+1, m-1\right\rangle .
\end{gathered}
$$

The matrix elements of angular momentum are trivially calculated in this basis; also the matrix elements of the electric dipole operator can be conveniently calculated by expressing it as a spherical tensor operator of rank 1 so that

$$
\begin{gathered}
e z=T_{0}^{(1)}, \quad e x=\frac{1}{\sqrt{2}}\left(T_{-1}^{(1)}-T_{1}^{(1)}\right), \\
e y=\frac{i}{\sqrt{2}}\left(T_{-1}^{(1)}+T_{1}^{(1)}\right) .
\end{gathered}
$$

We use the Wigner-Eckart theorem 


$$
\left\langle n l_{3} m_{3}\left|T_{m_{2}}^{\left(l_{2}\right)}\right| n^{\prime} l_{1} m_{1}\right\rangle=C_{m_{1} m_{2} m_{3}}^{l_{1} l_{2} l_{3}} \frac{\left\langle n l_{3}\left\|T^{\left(l_{2}\right)}\right\| n^{\prime} l_{1}\right\rangle}{\sqrt{2 l_{1}+1}} .
$$

Here, the first factor is the Clebsch-Gordan coefficient, and the second factor is the reduced matrix element which, in our case, is given by

$$
\left\langle n^{\prime}, l+1\|e \vec{r}\| n, l\right\rangle=\int_{0}^{\infty} d r \operatorname{er}^{3} R_{n^{\prime}, l+1}^{*}(r) R_{n l}(r) .
$$

The reduced matrix element is always nonvanishing, with $R_{n l}(r)$ being the radial wave function. We calculate the dipole matrix elements as

$$
\begin{gathered}
d_{31}^{x}=-\sqrt{\frac{(l-m+1)(l-m+2)}{2(2 l+2)(2 l+3)^{3}}}\left\langle n^{\prime}, l+1\|e \vec{r}\| n l\right\rangle, \\
d_{31}^{y}=i d_{31}^{x}, \quad d_{31}^{z}=0 .
\end{gathered}
$$

Using these expressions, we finally get

$$
\begin{aligned}
\nu= & \frac{\mu_{B}}{4}\left\langle n^{\prime}, l+1\|e \vec{r}\| n l\right\rangle(l-m+1) \\
& \times \sqrt{\frac{(l+m)(l-m+2)}{(l+1)(2 l+3)^{3}}}\left[\begin{array}{ccc}
-1 & -i & 0 \\
i & -1 & 0 \\
0 & 0 & 0
\end{array}\right]
\end{aligned}
$$

as the matrix which determines the orientation of the induced dipole moment. It should be noted that the matrix $\nu$ gives zero response to positively polarized em waves in accordance with the dipole selection rules. For our particular set of levels, we need negatively polarized em waves as they provide the photons with the correct helicity to satisfy the angular momentum conservation in the probe photon emission and absorption processes between the states 1 and 3. For negatively polarized waves, $\nu$ just reduces to a scalar.

It is worth noticing that the structure of the tensor $\nu$ resembles that of gyrotropic substances where both $\epsilon$ and $\mu$ are tensors, such as pure ferromagnetic metals and semiconductors. These were argued to be the most likely candidates to demonstrate left-handedness in the original paper by Veselago [9].

Further calculations require setting the polarizations of the coupling and the probe beams. To cause transitions between $|2\rangle$ and $|3\rangle$, the coupling beam polarization $\hat{\epsilon}_{d}$ has to have a component along the quantization direction $\hat{z}$. So let us take the coupling beam to propagate in the $x-y$ plane and be linearly polarized along $\hat{z}$. To cause transitions between states $|1\rangle$ and $|3\rangle$, the probe beam must have a polarization vector lying in the $x-y$ plane. Let us take it to be propagating along the $\hat{z}$ axis with polarization lying in the $x-y$ plane.

Then our general expression for the induced magnetic moment leads to

$$
\vec{\mu}=\gamma(\omega) E(\hat{x}-i \hat{y}),
$$

where $\omega$ now denotes the frequency of the probe beam and

$$
\begin{gathered}
\gamma(\omega)=\frac{\mu_{B}}{2 \hbar} \Omega_{2}^{*}\left\langle n^{\prime}, l+1\|e \vec{r}\| n l\right\rangle(l-m+1) \\
\times \sqrt{\frac{(l+m)(l-m+2)}{(l+1)(2 l+3)^{3}}} \frac{1}{Z}, \\
Z=4\left(i \Delta+\gamma_{31}\right)\left[i(\Delta-\delta)+\gamma_{21}\right]+\left|\Omega_{2}\right|^{2} .
\end{gathered}
$$

With this definition of $\gamma(\omega)$ we can extend our result to macroscopic electromagnetics of a gas with concentration $N$. In the spirit of the Clausius-Mossoti equation [6], we define the magnetization per unit volume as

$$
\vec{M}=N \gamma(\omega)\left(E+\frac{P}{3 \epsilon_{0}}\right)(\hat{x}-i \hat{y})=N \gamma(\omega)\left(1+\frac{\chi_{e}}{3}\right) E(\hat{x}-i \hat{y}) .
$$

Now we recall the Fourier transform of the curl equation for the electric field in Maxwell's equations. For a negatively polarized wave

$$
\vec{B}=\frac{1}{\omega} \vec{k} \times E(\hat{x}-i \hat{y})=\frac{i}{c} E(\hat{x}-i \hat{y}) .
$$

Combining Eqs. (30) and (31), we have

$$
\vec{M}=-i N \gamma(\omega) c\left(1+\frac{\chi_{e}}{3}\right) \vec{B} .
$$

Finally, by using the definitions $\vec{B}=\mu_{0}(\vec{H}+\vec{M})$ and $\vec{B}=\mu \vec{H}$, we get

$$
\mu_{r}(\omega)=\frac{1}{1+i \mu_{0} \gamma(\omega) c\left[1+\chi_{e}(\omega) / 3\right]}
$$

with $\mu_{r}=\mu / \mu_{0}$ is the relative (effective) permeability. We shall see that the combined effect of the electric and magnetic field components of the optical fields, as well as local field effects, lead to unusual light propagation regimes in particular at the EIT resonance frequency.

\section{RESULTS AND DISCUSSION}

We consider a gas of ${ }^{23} \mathrm{Na}$ atoms with $N=10^{24} \mathrm{~m}^{-3}$ to examine the case of dense media where the Lorentz-Lorenz local field corrections play a significant role and $N$ $=10^{12} \mathrm{~m}^{-3}$, for the case of a dilute gas where the local field effects are weak. Our results are presented in Fig. 2 for dense media and in Fig. 3 for a dilute gas.

We see that both the relative dielectric permittivity $\epsilon_{r}$ $=\epsilon / \epsilon_{0}$ and the relative magnetic permeability $\mu_{r}=\mu / \mu_{0}$ can become negative over a band of frequency $\sim 0.001 \gamma$. This allows the propagation of light through otherwise opaque media at high densities where electromagnetically induced transparency would not work. At resonance we find $\mu_{r}(0)=-0.69-i 0.11$ and $\epsilon_{r}(0)=-1.86+i 0.12$. It is natural to have transmission losses in our model, similar to other lefthanded structures, as they are unavoidable due to the Kramers-Kronig relations ensuring the causality in the system. On the other hand, theoretically it is not a trivial task to 

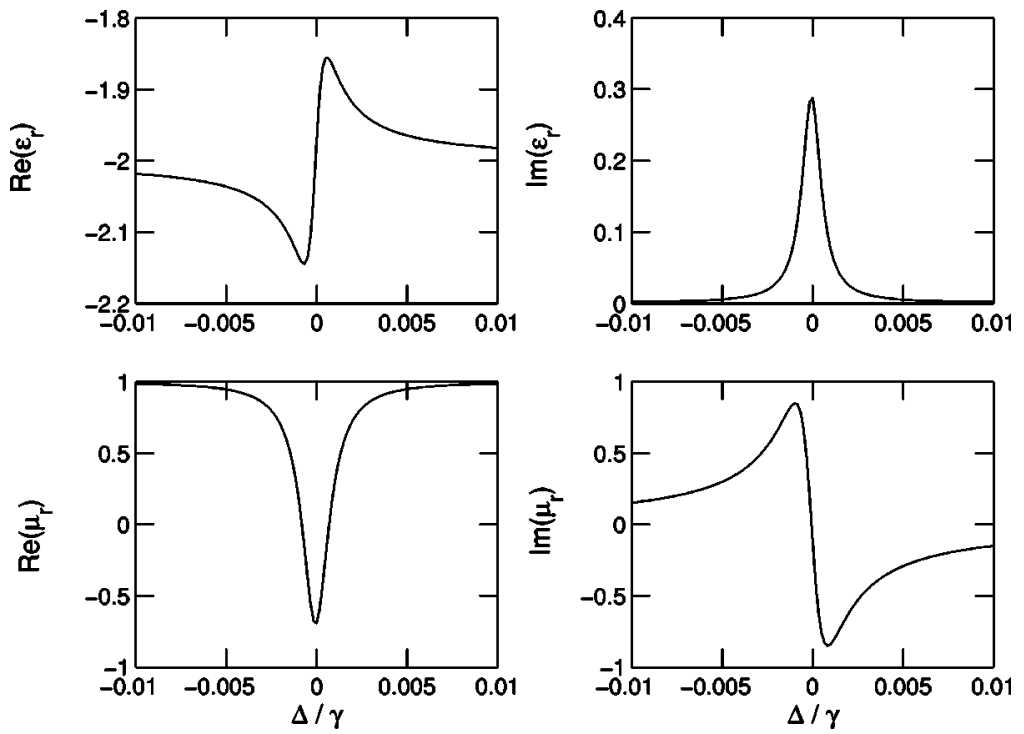

FIG. 2. Frequency dependence of the relative (effective) dielectric permittivity $\epsilon_{r}$ and the relative magnetic permeability $\mu_{r}$ of the dense gas of three-level atoms with $N=10^{24} \mathrm{~m}^{-3}, \lambda \sim 589 \mathrm{~nm}$, $\gamma \sim 10.06 \mathrm{MHz}, \gamma_{g e}=0.5 \gamma, \gamma_{g r} / 2 \pi \sim 10^{3} \mathrm{~Hz}$, and $\Omega_{2}=0.56 \gamma$. All axes are in dimensionless units. estimate the amount of losses [21-23] and to rigorously prove causality in left-handed materials $[24,25]$. We can give a simple and rough estimate by simply taking into account the imaginary part of the refractive index, which gives that after several micrometers the optical field will be damped by $\sim 33 \%$ due to linear absorption. At such length scales, our atomic system with the given densities may be found in a Bose-Einstein condensed state due to the interatomic interactions. Multiple scattering of photons as well as higher order many-body correlations may contribute in addition to the local field correction. Such effects are argued to be of about the same order as the local field correction [26-32]. It is an intriguing possibility that the present result of induced lefthandedness could improve and benefit from contributions arising from the quantum correlations in a dense BoseEinstein condensate or in a dense degenerate Fermi gas. In this paper, we will be content with limiting ourselves to classical gaseous media and hope to discuss the case of quantum gases elsewhere in detail.
In the dilute gas limit, we recover the usual behavior of the electric susceptibility under electromagnetically induced transparency conditions. The transparency region is in the valley between the twin peaks in the imaginary part of the electric susceptibility where the peaks correspond to the two dressed absorption lines, the Autler-Townes doublet [33]. The magnetic susceptibility exhibits a steep variation over a narrow band of frequencies in the vicinity of the resonance, while in magnitude the relative permeability remains close to unity for all $\Delta$. When $N \sim 10^{20}$, similar results to those shown in Fig. 3 are found where now $\mu_{r}$ varies between 1.002 and 0.9985 over $\Delta \in(-\gamma, \gamma)$.

In our numerical calculations, we estimate the dipole matrix element from the spontaneous emission rate $\gamma$ $\sim 10.06 \mathrm{MHz}$ using the relation $d_{31}=\sqrt{3 \gamma \hbar \epsilon_{0} \lambda^{3} / 8 \pi^{2}}$. Here $\lambda$ is the wavelength of the resonant probe transition which is $\lambda \sim 589 \mathrm{~nm}$. Typical values for $\gamma_{g e}=0.5 \gamma$ and $\gamma_{g r} / 2 \pi$ $\sim 10^{3} \mathrm{~Hz}$ are used. The Rabi frequency associated with the driving field is chosen to be $\Omega_{2}=0.56 \gamma$.
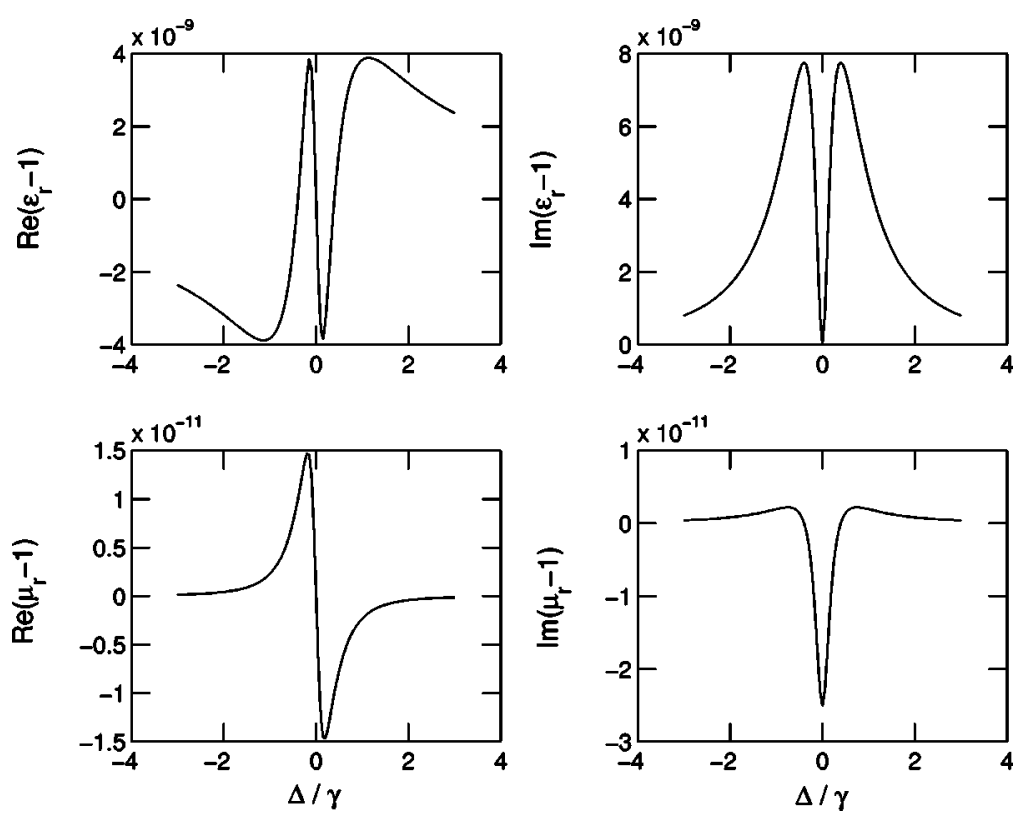

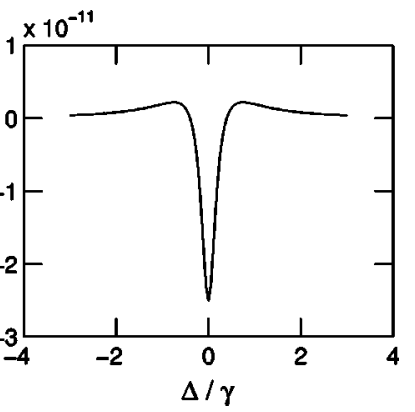

FIG. 3. Same as Fig. 2 but for the case of a dilute gas with $N=10^{12} \mathrm{~m}^{-3}$. Here, the electric susceptibility and the magnetic susceptibility are plotted as the relative permittivity and the permeability do not change appreciably from unity. 


\section{CONCLUSION}

In summary, we suggested a method for optical modification of magnetic permeability using a three-level scheme and derived the necessary conditions for its applicability. We found that it is in principle possible to electromagnetically induce left-handedness to a spatially homogeneous media. The major challenge we face is to have two levels separated at optical frequencies while having a nonvanishing magnetic dipole matrix element. Such level splittings require large external magnetic fields or should be engineered by other means such as external electric fields or spin-orbital couplings. One may also consider solid state systems, and try to utilize excitonic energy levels in solid state heterostructures to engineer three-level system satisfying the energy condition, or build quantum dots with a suitable spectrum. The predicted effect is fundamentally based upon the LorentzLorenz local field contribution in an electromagnetically induced transparent medium of three-level atoms with a nonvanishing dipole moment between lower levels. In the dense medium limit, in which the medium becomes opaque nor- mally with a negative dielectric constant, the presence of a magnetic dipole gives rise to a negative magnetic permeability so that the probe beam will still propagate within the otherwise optically thick dense medium for several micrometers before it is finally absorbed.

It should be emphasized that the presented method is applicable to spatially homogeneous media and does not need any spatial periodicity which is unavoidable in metamaterials. In the dilute medium limit, the value of permeability does not change from unity appreciably; however, in this case we observed that it demonstrates steep changes over a small band of frequency. Such a large gradient of permeability may affect the character of light propagation such as its group velocity and may serve an additional method to slow down or speed up the light.

\section{ACKNOWLEDGMENTS}

O.E.M. acknowledges useful discussions with A. Sennaroglu. M.O.O. thanks W. Ketterle for a preliminary discussion of the idea and encouragement.
[1] L.V. Hau, S.E. Harris, Z. Dutton, and C.H. Behroozi, Nature (London) 397, 594 (1999).

[2] D.F. Phillips, A. Fleischhauer, A. Mair, R.L. Walsworth, and M.D. Lukin, Phys. Rev. Lett. 86, 783 (2001).

[3] J.B. Pendry and D.R. White, Phys. Today 57(6), 37 (2004), and references therein.

[4] J.B. Pendry, Phys. Rev. Lett. 85, 3966 (2000).

[5] A. Mair, J. Hager, D.F. Phillips, R.L. Walsworth, and M.D. Lukin, Phys. Rev. A 65, 031802 (2002).

[6] J.D. Jackson, Classical Electrodynamics, 2nd ed. (Wiley, New York, 1975)

[7] M.O. Scully and M.S. Zubairy, Quantum Optics (Cambridge University Press, Cambridge, England, 1997).

[8] L.D. Landau, E.M. Lifshitz, and L.P. Pitaevskii, Electrodynamics of Continuous Media (Butterworth Heinemann, Oxford, 1998).

[9] V.G. Veselago, Usp. Fiz. Nauk 92, 517 (1964); [Sov. Phys. Usp. 10, 509 (1968)].

[10] D.R. Smith, W.J. Padilla, D.C. Vier, S.C. Nemat-Nasser, and S. Schultz, Phys. Rev. Lett. 84, 4184 (2000).

[11] J.B. Pendry, A.J. Holden, W.J. Stewart, and I. Youngs, Phys. Rev. Lett. 76, 4773 (1996).

[12] E. Cubukcu, K. Aydin, E. Ozbay, S. Foteinopoulou, and C.M. Soukoulis, Phys. Rev. Lett. 91, 207401 (2003).

[13] S.E. Harris, J.E. Field, and A. Imamoğlu, Phys. Rev. Lett. 64, 1107 (1990); K.J. Boller, A. Imamoğlu, and S.E. Harris, ibid. 66, 2593 (1991); M.O. Scully, Phys. Rep. 219, 191 (1992); S.E. Harris, Phys. Today 50(7), 36 (1997), and references therein.

[14] G. Morigi and G.S. Agarwal, Phys. Rev. A 62, 013801 (2000).

[15] C.M. Bowden and J.P. Dowling, Phys. Rev. A 47, 1247 (1993).
[16] R. Binder and M. Lindberg, Phys. Rev. B 61, 2830 (2000)

[17] H. Schmidt, K.L. Chapman, A.C. Gossard, and A. Imamoğlu, Appl. Phys. Lett. 70, 3455 (1997).

[18] D.E. Nikonov, A. Imamoglu, and M.O. Scully, Phys. Rev. B 59, 012212 (1999).

[19] M.S. Bigelow, N.N. Lepeshkin, and R.W. Boyd, Phys. Rev. Lett. 90, 113903 (2003).

[20] W.W. Chow, H.C. Schneider, and M.C. Phillips, Phys. Rev. A 68, 053802 (2003).

[21] N. Garcia and M. Nieto-Vesperinas, Opt. Lett. 27, 885 (2002).

[22] P. Markoš, I. Rousochatzakis, and C.M. Soukoulis, Phys. Rev. E 66, 045601 (2002).

[23] C.G. Parazzoli, R.B. Greegor, K. Li, B.E. C. Koltenbah, and M. Tanielian, Phys. Rev. Lett. 90, 107401 (2003).

[24] R.W. Ziolkowski and A.D. Kipple, Phys. Rev. E 68, 026615 (2003).

[25] T. Koschny, P. Markoš, D.R. Smith, and C.M. Soukoulis, Phys. Rev. E 68, 065602 (2003).

[26] K.V. Krutitsky, F. Burgbacher, and J. Audretsch, Phys. Rev. A 59, 1517 (1999).

[27] H. Wallis, Phys. Rev. A 56, 2060 (1997).

[28] O. Morice, Y. Castin, and J. Dalibard, Phys. Rev. A 51, 3896 (1995).

[29] J. Ruostekoski and J. Javanainen, Phys. Rev. A 55, 513 (1997).

[30] J. Ruostekoski and J. Javanainen, Phys. Rev. A 56, 2056 (1997).

[31] M. Fleischhauer, and S.F. Yelin, Phys. Rev. A 59, 2427 (1999).

[32] K.V. Krutitsky, F. Burgbacher, and J. Audretsch, Laser Phys. 10, 15 (2000).

[33] S.H. Autler and C.H. Townes, Phys. Rev. 100, 703 (1955). 